\title{
Alimentación de la ascidia Ecteinascidia turbinata (Ascidiacea: Perophoridae) en dos áreas de manglar de Cuba
}

\author{
Aida Hernández-Zanuy ${ }^{1}$, Alida García-Cagide ${ }^{1}$, Nilda Borrero ${ }^{1}$ \& José Luis Carballo ${ }^{2}$ \\ 1 Instituto de Oceanología del Ministerio de Ciencia Tecnología y Medio Ambiente de Cuba. Ave. 1era y 186 No. \\ 18406, Playa, La Habana, Cuba; aidah@oceano.inf.cu \\ 2 Instituto de Ciencias del Mar y Limnología, UNAM. Estación Mazatlán. Apartado Postal 811. Mazatlán 82000. \\ México.FAX: 5269826133; carballo@ola.icmyl.unam.mx
}

Recibido 10-VII-2002. Corregido 24-XI-2006. Aceptado 06-XII-2006.

\begin{abstract}
Diet of the ascidian Ecteinascidia turbinata (Ascidiacea: Perophoridae) in two mangrove areas of Cuba. Stomach contents of 88 zooids of Ecteinascidia turbinata Herdman 1880 and the qualitative and quantitative composition of phytoplankton in the water column were studied in Santa Fe (North Coast of Havana) and Punta del Este (SW of Cuba). We identified 59 microalgal species and four tintinnids in the stomachs. Cell size was $75-165 \mu \mathrm{m}$ in length and 2-105 $\mu \mathrm{m}$ in width. There were not significant differences in microalgal diversity in the water column in the two locations. In both locations, the diatoms had the largest number of species and individuals in stomachs and water. In Santa Fe, dinoflagellate biomass was larger in water and stomach contents, while in Punta del Este the contribution of each group to the stomach content was similar to that of the water column. This species filters in a constant and irregular way during the day, independently of food availability. Rev. Biol. Trop. 55 (2): 499-507. Epub 2007 June, 29.
\end{abstract}

Key words: Ecteinascidia turbinata, ascidian, feeding, phytoplankton, mangroves.

En las ascidias se encuentran los principales grupos tróficos del bentos marino: filtradoras de fitoplancton y partículas en suspensión (Millar 1971), filtradoras de partículas sedimentarias (Diehl 1957) y carnívoras que capturan sus presas para alimentarse (Monniot y Monniot 1968). La velocidad de filtración depende del tamaño y complejidad de las branquias y en condiciones de laboratorio se determinó que pueden filtrar, de forma irregular y continua durante las 24 horas del día, hasta más de $6000 \mathrm{ml} / \mathrm{h} / \mathrm{g}$ de peso seco del organismo, con un $85 \%$ de asimilación del alimento (Fiala-Médioni 1974).

Ecteinascidia turbinata Herdman 1880 es una ascidia colonial de cuyas características alimentarias no se tiene información a pesar de que en los últimos años se han estudiado diferentes aspectos de su biología (Young 1986, Bingham y Young 1991, Vazquez y Young 1996, Carballo et al. 1997, 2000, Carballo 2000, HernándezZanuy et al. 2000) debido a la importancia de la especie como fuente de sustancias antitumorales (Rinehart et al. 1990).

El presente trabajo es parte de un proyecto dedicado al estudio de la biología, ecología y métodos de producción de E. turbinata en los manglares cubanos y tiene como objetivo conocer el espectro alimentario de la especie y su relación con la composición y la disponibilidad de alimento en dos áreas de manglar ubicadas al sur y norte de la región occidental de Cuba.

\section{MATERIALES Y MÉTODOS}

Se realizaron dos muestreos en dos áreas de manglar de la región occidental de Cuba. El primero se realizó en el mes de enero de 1995 en un pequeño manglar de aproximadamente 
$25 \mathrm{~m}$ de largo ubicado en una laguna costera del poblado de Santa Fe (23 $05^{\prime}$ N, $\left.82^{\circ} 30^{\prime} \mathrm{W}\right)$, en el litoral norte de la Ciudad de la Habana (Fig. 1). El segundo se realizó en marzo del mismo año en un canal de $15 \mathrm{~m}$ de ancho y aproximadamente $300 \mathrm{~m}$ de largo, bordeado de mangle y con un pequeño cayo central, ubicado en el área del manglar de Punta del Este $\left(21^{\circ}\right.$ $33^{\prime} \mathrm{N}, 82^{\circ} 32^{\prime} \mathrm{W}$ ), Isla de la Juventud (Fig. 1). En ambas localidades, la profundidad varía entre 0.5 y $1.5 \mathrm{~m}$ y el intercambio de agua con el océano es escaso.

En Santa Fe se marcaron tres colonias y cada cuatro horas durante 24 horas, se recolectaron cinco zooides de forma aleatoria de cada colonia. En Punta del Este se utilizaron dos colonias, con la misma metodología de colecta pero el muestreo tuvo una duración de 48 horas. Los zooides se preservaron en formaldehido al $4 \%$ diluido con agua de mar y neutralizado con tetraborato de sodio. En ambas estaciones se tomaron muestras de agua a $25 \mathrm{~cm}$ de profundidad cada cuatro horas con botellas oscuras de un litro de capacidad. Estas se fijaron con lugol ácido y se procesaron según la técnica descrita por Popowski y Borrero (1989) para el estimado de la concentración fitoplanctónica (cél./ml). El estimado de carbono para calcular la biomasa fitoplanctónica $\left(\mathrm{mgC} / \mathrm{m}^{3}\right)$ se realizó por el método de Smayda (1965).

Bajo un microscopio estereo, a cada zooide se le separó el tracto digestivo del resto del cuerpo y se colocó sólo el contenido estomacal (excluyendo esófago e intestino) en un volumen conocido de una solución de agua de mar con lugol ácido. En total se analizaron 88 estómagos (36 de las muestras tomadas en Santa Fe y 52 de Punta del Este). La identificación se realizó hasta el taxón más bajo posible.

Debido a que los contenidos estomacales fueron preservados en formaldehido y se ha comprobado que este fijador destruye los flagelados después de 45 días de fijadas las muestras (Popowski y Borrero1989), no se tuvo en cuenta la presencia de este grupo en el análisis cuantitativo de los contenidos estomacales y la columna de agua, para poder comparar los mismos.
Para establecer las correlaciones de la densidad y biomasa de microalgas en los estómagos con la densidad y biomasa celular en la columna de agua, se utilizó la prueba de Spearman (95\% de confiabilidad). El estado trófico del agua se caracterizó por la escala de Koblentz-Mishke y Vedernikov (1977) y para la diversidad de especies se empleó el índice de Shannon y Weaver (1963) expresado en bitios. La altura de la marea en Punta del Este se tomó de las Tablas de Marea 1994 y 1995 y para Santa Fe se utilizaron los datos del mareógrafo del Instituto de Oceanología.

\section{RESULTADOS}

Composición del espectro alimentario: $\mathrm{Se}$ identificaron 59 especies de microalgas y cuatro tintínidos en los contenidos estomacales de E. turbinata recolectados en las dos localidades (Cuadro 1). El tamaño celular varió entre 75 y

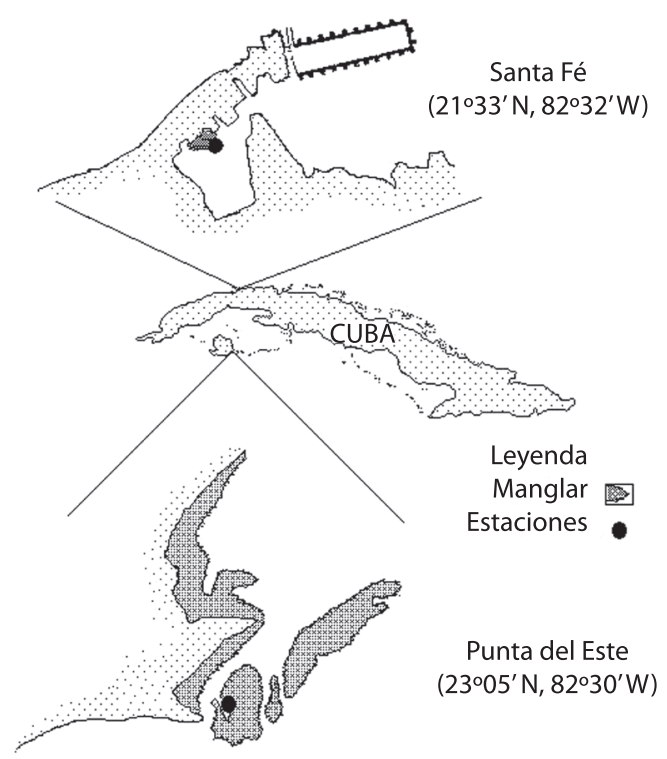

Fig. 1. Ubicación geográfica de las áreas de estudio (Santa Fe: $23^{\circ} 05^{\prime} \mathrm{N}, 82^{\circ} 30^{\prime} \mathrm{W}$; Punta del Este: $21^{\circ} 33^{\prime} \mathrm{N}, 82^{\circ}$ 32' W).

Fig. 1. Geographical location of the study areas (Santa Fe: $23^{\circ} 05^{\prime} \mathrm{N}, 82^{\circ} 30^{\prime} \mathrm{W}$; Punta del Este: $21^{\circ} 33^{\prime} \mathrm{N}, 82^{\circ}$ 32 ' W). 
CUADRO 1

Composición de especies en el agua y en los contenidos estomacales de Ecteinascidia turbinata en Santa Fe y Punta del Este, dos áreas de manglar de Cuba

TABLE 1

Species composition in the water and stomachs of Ecteinascidia turbinata in Sante Fe and Punta del Este, two mangrove areas of $\mathrm{Cuba}$

Grupos y Especies

\section{Diatomeas}

Amphora laevis

Amphora sp.

Amphora sp. 1

Amphiroa sp.

Cocconeis sp.

Cocconeis $\mathrm{sp}_{{ }_{1}}$

Cocconeis $\mathrm{sp}_{2}$

Coscinodiscus sp.

Cymbella sp.

Diploneis cabro

Diploneis fusca

Diploneis smithie

Diploneis sp.

Diploneis weissflagi

Grammathophora marina

Licmophora abreviata

Licmophora gracilis

Mastogloia sp.

Melosira sulcata

Navicula cancellata

Navicula lancellata

Navicula $\mathrm{sp}_{{ }_{1}}$

Navicula $\mathrm{sp}_{2}$

Navicula $\mathrm{sp}_{3}$

Navicula $\mathrm{sp}$.

Navicula $\mathrm{sp}_{{ }_{5}}$

Navicula $\mathrm{sp}_{6} 6$

Navicula $\mathrm{sp}_{7}$

Navicula $\mathrm{sp}_{.}$

Navicula $\mathrm{sp}_{.}$

Navicula $\mathrm{sp}_{\cdot 10}$
Santa Fe

Agua

X

Estómago

X

X

X

X

X

X

X

X

X

X

X

X

X

$\begin{array}{ll}X & X \\ X & X \\ X & X\end{array}$

X

X $\quad$ X

X

X $\mathrm{X}$

$\mathrm{X} \quad \mathrm{X}$

$\mathrm{X} \quad \mathrm{X}$

X X

$\mathrm{X}$

X

X
X

X

$\mathrm{X}$

$\mathrm{X}$

$\mathrm{X}$

$\mathrm{X}$

X

X

X

X

$\mathrm{X}$

$x$

X$$
\mathrm{X}
$$$$
\mathrm{X}
$$

X

$\mathrm{X}$

X

X

X

X

X

X

X

X

X

X

X

X 
Nitzchia longissima

Nitzchia sp.

Pleurosigma - Gyrosigma

Rabdonema adriaticum

Striatella interrupta

Striatella unipunctata

Synedra sp.

Synedra sp.2

Synedra undulata

Synidia sp.

Triceratium sp.

\section{Dinoflagelados}

Dinophysis caudatus

Dinophysis sp.

Gonyaulax sp.

Gymnodinium $\mathrm{sp}_{1}$

Gymnodinium $\mathrm{sp}_{2}$

Gymnodinium $\mathrm{sp}_{3}$

Protoperidinium sp.

Prorocentrum lima

Prorocentrum micans

\section{Cloroficeas}

Poropila dubia

X $\mathrm{X}$

X

$\mathrm{X}$

X $\mathrm{X}$ X

$\mathrm{X}$

X

X

X

X

X

X

X

X

X

X

X

X

X

X

X

\section{Cianobacterias}

Cianobacterias sp.

Cianobacterias $\mathrm{sp}_{{ }_{1}}$

Oscillatoria willi

X

Oscillatoria $\mathrm{sp}_{{ }_{1}}$

Oscillatoria $\mathrm{sp}_{2}$

Spirulina major

\section{Tintinidos}

Eutintinnus sp.

Favela campanula

Tintinopsis beroide

Tintinopsis sp.
X

X

$\mathrm{X} \quad \mathrm{X}$

X

$\begin{array}{lll} & X & X \\ X & X & X \\ X & & \\ & & X \\ & & X \\ & X & X \\ & X & X\end{array}$

X

X

X $\mathrm{X}$
X

X

$\mathrm{X}$

$\mathrm{X}$

X

X

$\mathrm{X}$

$\mathrm{X}$

$\mathrm{X}$

$\mathrm{X}$

X

X

$\mathrm{X}$

$\mathrm{X}$

X

X

X

X

X

X$$
\mathrm{X}
$$

x$$
\mathrm{X}
$$$$
\text { X }
$$$$
\text { X }
$$

x

X

X

$x$


$165 \mu \mathrm{m}$ de largo y de 2 a $105 \mu \mathrm{m}$ de ancho. Las microalgas fueron en su mayoría especies del microfitobentos, aunque también se encontraron especies planctónicas. El número de especies de tintínidos y de los diferentes grupos de microalgas, fue mayor en los estómagos que en el agua (Cuadro 1). Las diatomeas con 42 especies, y en particular el género Navícula, fueron el grupo más abundante en los estómagos de las dos localidades (19 especies en Santa Fe y 35 en Punta del Este). Los dinoflagelados (nueve especies) fueron el segundo grupo más importante. El resto de los grupos presentes en la dieta de esta ascidia estuvieron pobremente representados. Se observaron ciliados, Oikopleura, copépodos y nauplios en el $2 \%$ de los sacos branquiales de los zooides, pero no en los contenidos estomacales.

Las diatomeas representaron el $80 \%$ de la abundancia celular microalgal en los contenidos estomacales y el $70 \%$ del total en la columna de agua, en ambas localidades (Fig. 2). Sin embargo, cuando se analizó la biomasa fitoplanctónica, los dinoflagelados aportaron el
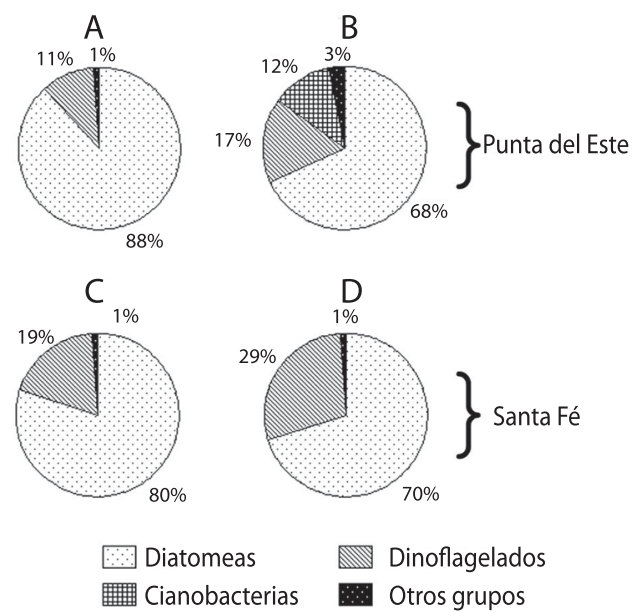

Fig. 2. Porcentajes de los grupos de fitoplancton encontrados en los estómagos de Ecteinascidia turbinata (A y C) y en la columna de agua (B y D); en Punta del Este (A y B) y en Santa Fe (C y D).

Fig. 2. Percent distribution of phytoplancton groups in the stomachs of Ecteinascidia turbinata (A and C) and in the column of water (B and D); in Punta del Este (A and B) and in Santa Fe (C and D).
$56 \%$ del total en los contenidos estomacales en Punta del Este y el 84 \% en Santa Fe (Fig. 3 A, C). En la columna de agua, estos organismos representaron el $89 \%$ de la biomasa en Santa $\mathrm{Fe}$, pero en Punta del Este los porcentajes de los diferentes grupos de microalgas fueron semejantes (Fig. 3 B, D).

No se encontraron diferencias significativas entre la diversidad de especies de microalgas en la columna de agua, en ambas localidades. De acuerdo a la escala de Koblentz-Mishke y Vedernikov (1977), tanto por el número de células como por la biomasa, el agua en estas dos áreas presentó características de mesotróficas a eutróficas.

Ritmo diario de alimentación: En los dos muestreos realizados, fue notable la variabilidad individual en las concentraciones de microalgas en los contenidos estomacales de los zooides de cada colonia (independiente del largo del zooide) y de las colonias entre sí. Durante todas las horas la cantidad de células vivas siempre fue superior a la de células
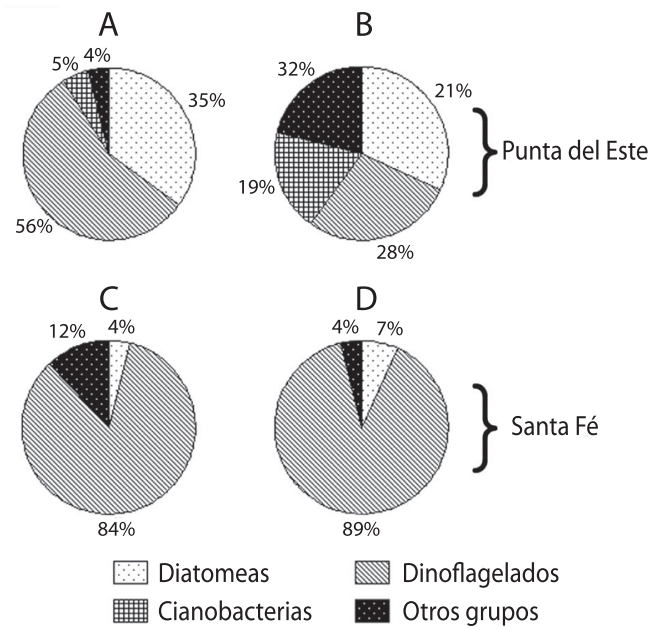

Fig. 3. Biomasa por grupos de fitoplancton encontrado en los estómagos de Ecteinascidia turbinata (A y C) y en la columna de agua (B y D); en Punta del Este (A y B) y en Santa Fe (C y D).

Fig. 3. Biomass for phytoplancton groups in the stomachs of Ecteinascidia turbinata (A and $\mathrm{C}$ ) and in the water column (B and D); in Punta del Este (A and B) and in Santa $\mathrm{Fe}(\mathrm{C}$ and $\mathrm{D})$. 
muertas en los estómagos lo que indica que los zooides filtran activamente durante las 24 horas del día.

Al comparar las fluctuaciones en la amplitud de las mareas con la densidad y la biomasa de microalgas en la columna de agua y en los estómagos durante el ciclo de 24 horas en la estación de Santa Fe, y el ciclo de 48 horas en Punta del Este (Fig. 4 y 5, respectivamente) se encontró que la abundancia microalgal en la columna de agua varió de acuerdo al cambio mareal, pero no se encontró correlación entre
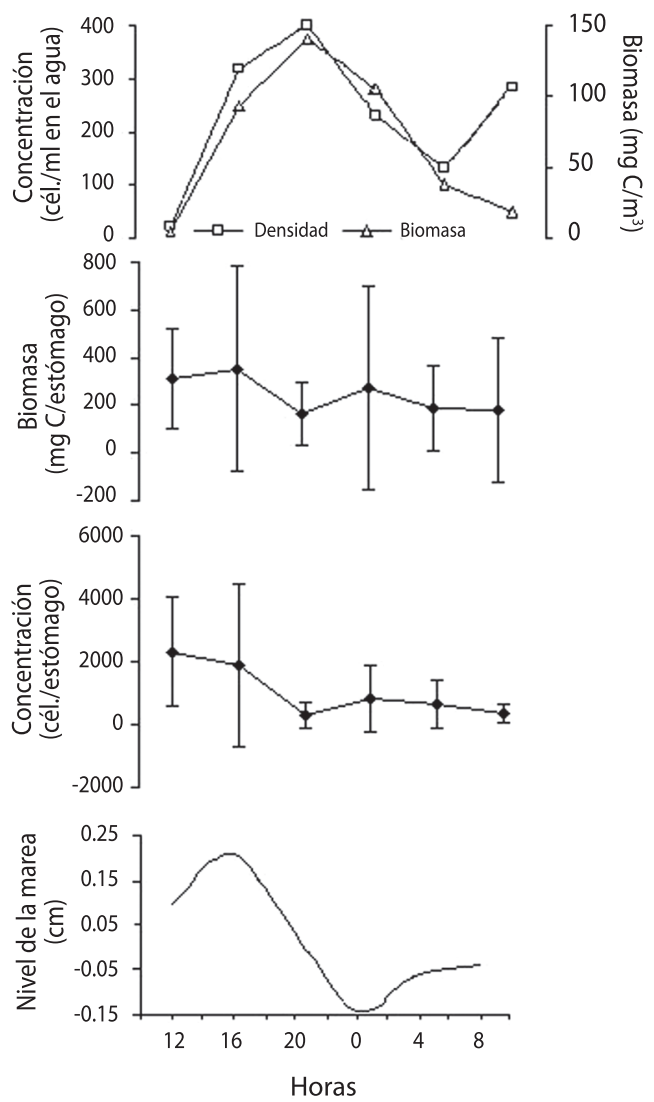

Fig. 4. Variación de la concentración y biomasa de fitoplancton en el agua y en los estómagos de Ecteinascidia turbinata y su relación con la amplitud de la marea, durante un ciclo de 24 h, en Santa Fe.

Fig. 4. Variation of the concentration and phytoplancton biomass in the water and in the stomachs of Ecteinascidia turbinata and their relationship with tide range, during a 24 $\mathrm{h}$ cycle, in Santa Fe. la densidad y biomasa celular en los estómagos $y$ en el agua.

\section{DISCUSIÓN}

Según los resultados obtenidos E. turbinata se alimenta casi exclusivamente de
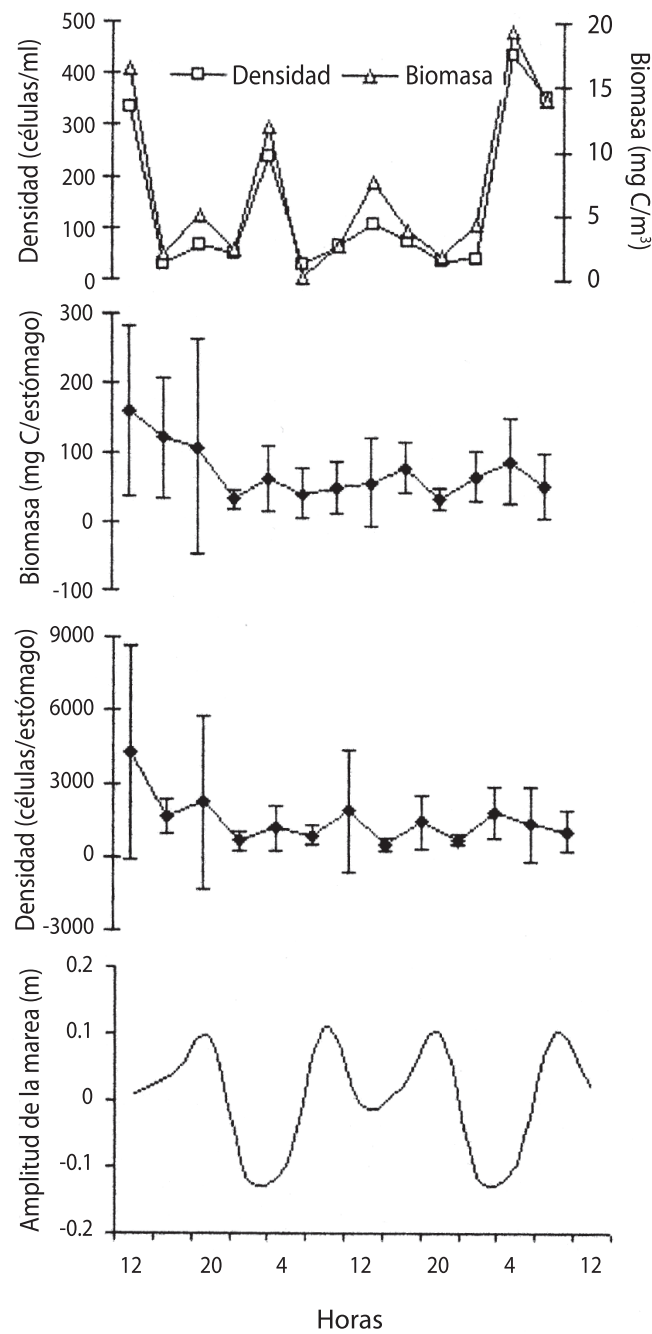

Fig. 5. Variación de la concentración y biomasa de fitoplancton en el agua y en los estómagos de Ecteinascidia turbinata y su relación con la amplitud de la marea, durante un ciclo de 48 h, en Punta del Este.

Fig. 5. Variation of the concentration and phytoplancton biomass in the water and in the stomachs of Ecteinascidia turbinata and their relationship with tide range, during a 48 h cycle, in Punta del Este. 
microalgas a pesar de que el tamaño del sifón inhalante permite la entrada de organismos de mayor talla, en especial copépodos, los cuales, al igual que otros crustáceos, son huéspedes habituales de la cavidad branquial en algunas ascidias (Humes y Stock 1973) debido a la no existencia de una defensa efectiva contra la entrada de estos organismos (Millar 1971).

Con relación a las partículas alimenticias de menor talla (bacterias, detritus, etc.) que también penetran al tracto digestivo de la ascidia, no se puede descartar que estas sean utilizadas en la alimentación ya que el método empleado no permite cuantificar estas fracciones; sin embargo, sobre la eficiencia de retención de las partículas alimenticias en ascidias, se ha planteado que ésta depende de la porosidad del mucus secretado por el endostilo (Jørgensen y Goldberg 1953) y existen diferentes criterios en cuanto a la retención de partículas muy pequeñas. Randløv y Riisgård (1979) demostraron que partículas entre 2 y $3 \mu \mathrm{m}$ de diámetro pueden ser completamente retenidas por cuatro especies de ascidias solitarias mientras que Stuart y Klumpp (1984) observaron que la especie Pyura stolonifera retenía todas las partículas mayores de $0.6 \mu \mathrm{m}$ con aproximadamente un $100 \%$ de eficiencia.

Las tallas de los organismos identificados en los contenidos estomacales de E. turbinata en el presente estudio indican que organismos mayores de $2 \mu \mathrm{m}$ y hasta $165 \mu \mathrm{m}$ pueden ser retenidos por la capa mucosa del endostilo e incorporarse al estómago. Resultados similares han sido encontrados en el ostión Crassostrea rhizophorae, especie muy abundante entre la epifauna asociada a la raíz de mangle en Cuba, que ingiere partículas entre 1 y $98 \mu \mathrm{m}$ de diámetro (Spittler et al. 1989).

Los componentes básicos del espectro alimentario de E. turbinata (diatomeas y dinoflagelados) fueron considerados por López Baluja et al. (1980) como las principales comunidades de microalgas del Archipiélago Cubano aunque posteriormente Popowski y Borrero (1989), al utilizar otras técnicas de fijación y análisis de las muestras, demostraron que los flagelados constituían el 70\% de la comunidad fitoplanctónica en el Golfo de Batabanó (área donde se encuentra ubicada la localidad de Punta del Este).

La proporción de los grupos principales de microalgas en la columna de agua, en las dos localidades estudiadas, coincide con la encontrada en los contenidos estomacales. Aunque por limitaciones del método de fijación empleado no se tuvieron en cuenta los flagelados en el análisis cuantitativo, la proporción de flagelados en la columna de agua fue del $54 \%$ en Santa Fe y $38 \%$ en Punta del Este, por lo cual no se puede descartar que este grupo forme parte de la dieta de E. turbinata.

Jørgensen (1955) planteó que las ascidias pueden ingerir lo que necesitan. Según Kott (1989), el alimento obtenido por un organismo filtrador está relacionado con la concentración del alimento presente en el agua, el tiempo empleado en la filtración y la capacidad de filtración individual. En el caso de E. turbinata no se pudo diferenciar un ritmo de alimentación en el medio natural. Resultados semejantes obtuvo Fiala-Médioni (1974) en cuatro especies de ascidias, al suministrarles una concentración constante de alimento durante 24 horas, y Stuart y Klumpp (1984) encontraron que la tasa de filtración en Pyura stolonifera eran independiente de la concentración del alimento.

Al parecer E. turbinata consume organismos fitoplantónicos y del microbentos que son arrastrados por las mareas desde la zona de manglar que emerge en marea baja. En esta zona se acumula una gran cantidad de materia orgánica y de taninos y hay una microbiota abundante la cual está encargada de los procesos de remineralización. Se conoce que existen también dinoflagelados bioluminiscentes asociados a los manglares, con actividades biológicas notables las que han sido asociadas a la presencia de sustancias húmicas en este biotopo (Cintron y Schaeffer-Noveli 1983). Aunque los dinoflagelados de los géneros Dynophysis, Prorocentrum y Gymnodinium, encontrados en los estómagos de E. turbinata, están considerados como tóxicos (Hallegraeff 1995) en nuestras aguas no existe ningún reporte de toxicidad. Sin embargo, teniendo en cuenta la elevada concentración de células que llega a 
encontrarse en un zooide, y que las colonias de esta especie pueden llegar a tener miles de zooides no puede descartarse alguna participación de estos organismos en la notable bioactividad de los extractos de esta especie que son verdaderos almacenes de microalgas. Estudios posteriores esclarecerán las posibles relaciones entre la alimentación de un organismo filtrador y la actividad biológica de sus extractos.

\section{AGRADECIMIENTOS}

El presente trabajo fue realizado gracias al financiamiento de la Agencia de Medio Ambiente del Ministerio de Ciencia Tecnología y Medio Ambiente de Cuba y la colaboración de la Delegación del CITMA de la Isla de la Juventud. Agradecemos a Pedro Alcolado Menéndez la revisión crítica de este artículo, a Macario Esquivel su participación en los muestreos y en el procesamiento de los datos de los estómagos, y a Magalys Sánchez su apoyo en la elaboración de los gráficos.

\section{RESUMEN}

Se estudiaron los contenidos estomacales de 88 zooides de Ecteinascidia turbinata Herdman 1880 y la composición cualitativa y cuantitativa del fitoplancton en la columna de agua en Santa Fe (Litoral Norte de la Habana) y Punta del Este (SW de Cuba). En el contenido estomacal de la ascidia se identificaron 59 especies de microalgas y cuatro tintínidos. El tamaño de las células varió entre 75 y $165 \mu \mathrm{m}$ de largo y de 2 a $105 \mu \mathrm{m}$ de ancho. No se encontraron diferencias significativas en la diversidad de especies de microalgas en la columna de agua de las dos localidades. Las diatomeas tuvieron la mayor cantidad de especies y el mayor número de individuos, tanto en los estómagos como en la columna de agua en ambas localidades. La biomasa de dinoflagelados en Santa Fe fue mayor en agua y estómagos. En Punta del Este el aporte de cada grupo al contenido estomacal es similar al encontrado en la columna de agua. Esta especie filtra de forma constante e irregular durante las 24 horas del día, independientemente de la disponibilidad de alimento.

Key words: Ecteinascidia turbinata, ascidia, alimentación, fitoplancton, manglar.

\section{REFERENCIAS}

Bingham, B.L. \& C.M. Young. 1991. Influence of sponges on invertebrate recruitment: a field test of allelopathy. Mar. Biol. 109: 9-26.

Carballo, J.L., S. Naranjo, B. Kukurtzü \& A. HernándezZanuy. 1997. Estudio de la población de Ecteinascidia turbinata Herdman 1880 (Ascidiacea:Perophoridae) en la isla de Formentera (Mar Mediterráneo. España): Distribución, densidad y crecimiento. Cienc. Mar. UAS 15: 7-15.

Carballo, J.L., S. Naranjo, B. Kukurtzü, F. De La Calle \& A. Hernández-Zanuy. 2000. Production of Ecteinascidia turbinata (Ascidiacea: Perophoridae) for obtaining anticancer compounds. J. World Aquac. Soc. 31: 481-490.

Carballo, J.L. 2000. Larval ecology of an ascidian tropical population in a mediterranean enclosed ecosystem. Mar. Ecol. Prog. Ser. 195: 159-167.

Cintron, G.Y. \& Schaeffer-Noveli 1983. Mangrove Forest: Ecology and response to natural and man induced stressors. Report of a Workshop held at West Indies Laboratory, St.Croix, US. UNESCO Rep., 133 p.

Diehl, M. 1957. Die okologie dei Ascidie Styela coriacea in der Kieler Bucht. Kieler Meeresforsch 13:59-68.

Fiala-Médioni, A. 1974. Filter-feeding Ethology of Benthic Invertebrates (Ascidians). II. Variations in Filtration and Digestion Rates as a Function of Spercies. Mar. Biol. 28: 99-206.

Hallegraeff, G.M. 1995. Harmful Algal Blooms: A global overview, p. 1-22. In G.M.. Hallegraff, D.M. Anderson \& A.D. Cembella (eds.). IOC Manuals and Guides, No.33. UNESCO, París.

Hernández-Zanuy, A., A. García-Cagide, M. Esquivel \& A. Blanco. 2000. Reproducción y desarrollo de Ecteinascidia turbinata (Ascidiacea: Perophoridae) en Cuba. Rev. Biol. Trop. 48:193-199.

Humus, A.G. \& H. Stock. 1973. A revision of the family Lichomolgidae Korsman, 1877, cyclopoid copepods, mainly associated with marine invertebrates. Smithson. Contr. Zool. 127:1-368.

Jørgensen, C.B. 1955. Quantitative aspects of filter-feeding in invertebrates. Biol Rev. 30:391-454.

Jørgensen, C.B. \& E.D. Goldberg 1953. Particle filtration in some ascidians and lamellibranches. Bull. Mar. Biol. Lab., Wood Hole, 105: 477-489. 
Koblentz-Mishke, O. I. \& V. I. Vedermikov. 1977. Producción primaria, p. 86-97. In Biología del Océano. Producción biológica (en ruso) Vol. 2, Nauka, Moscú.

Kott, P. 1989. Form and function in the ascidiacea. Bull Mar. Scien. 45:253-276.

López Baluja, L., N. Borrero \& G. Popowski. 1980. Distribución cualitativa y cuantitativa del fitoplancton en la plataforma de Cuba, Instituto de Oceanología., La Habana, 48 pp.

Millar, R.H. 1971. The Biology of Ascidians, p. 1-100. In F.S. Russell \& M. Yonge (eds.). Advances in Marine Biology, Vol. 9, Academic Press, Nueva York, Nueva York, EEUU.

Monniot, C. \& F. Monniot, 1968. Les Ascidies de grande profundeur récoltées par le navire oceanographique américain «Atlantis II ». Bull. Inst. Océano. Monaco 67: 1-48.

Popowski, G. \& N. Borrero. 1989. Utilización de fijadores en la conservación de flagelados y su eficacia en la determinación de la concentración del fitoplancton en el Golfo de Batabanó, Cuba. Rep. Invest. Inst. Oceanol. 11: 1-8.

Randløv, A. \& H.U. Riisgård. 1979. Efficiency of particle retention and filtration rate in four species of ascidians. Mar. Ecol. Prog. Ser. 1:55-59.
Rinehart, K.H., T.G. Holt, N.L. Fregeau, J.G. Stroh, P.A. Keiper, F.Sun, L.H. Li \& D.G. Martin. 1990. Ecteinascidins 729, 743, 745, 579A, 759B, and 770 potent antitumor agents from the Caribbean tunicate Ecteinascidia turbinata. J. Org. Chem. 55: 452-455.

Shannon, C.E. \& W. Weaver. 1963. The mathematical theory of communication. Univ. Illinois, Urbana, Illinois, EEUU. 117 pp.

Smayda, T.J. 1965. A quantitative analysis of the phytoplancton of the Gulf of Panamá. On the relationship beteween $\mathrm{C}^{14}$ assimilation and the diatom standing crop. Bull. Inter. Amer. Trop. Tuna Comm. 9: 465531.

Spither, P., A. de la Cruz \& J. Rodríguez. 1989. La selección del tamño de las partículas alimenticias por el ostión Crassostrea rhizophorae. Rev. Invest. Mar. 10:63-70.

Stuart, V. \& D.W. Klumpp. 1984. Evidence for foodresource partitionning by kelp-bed filter feeders. Mar. Ecol. Prog. Ser. 16:27-37.

Vazquez, E. \& C.M. Young. 1996. Responses of compound ascidian larvae to haloclines. Mar. Ecol. Prog. Ser. 133:179-190.

Young, C. M. 1986. Direct observations of field swiming behavior in larvae of the colonial ascidians Ecteinascidia turbinata. Bull. Mar. Scien. 39:279- 289. 
